\title{
Kernos
}

Revue internationale et pluridisciplinaire de religion grecque antique

$26 \mid 2013$

Varia

\section{Divination et magie}

Remarques sur les papyrus grecs de l'Égypte gréco-romaine

\section{Emilio Suárez de la Torre}

\section{OpenEdition \\ 1 Journals}

Édition électronique

URL : http://journals.openedition.org/kernos/2210

DOI : $10.4000 /$ kernos.2210

ISSN : 2034-7871

\section{Éditeur}

Centre international d'étude de la religion grecque antique

\section{Édition imprimée}

Date de publication : 10 octobre 2013

Pagination : 157-172

ISSN : 0776-3824

\section{Référence électronique}

Emilio Suárez de la Torre, « Divination et magie », Kernos [En ligne], 26 | 2013, mis en ligne le 31 octobre 2015, consulté le 02 mars 2021. URL : http://journals.openedition.org/kernos/2210 ; DOI : https://doi.org/10.4000/kernos.2210 


\section{Divination et magie}

\section{Remarques sur les papyrus grecs de l'Égypte gréco-romaine ${ }^{1}$}

Résumé : L'article étudie plusieurs aspects des sortilèges divinatoires des papyrus magiques de l'Égypte romaine. D'abord, il soutient que les limites entre la magie et la divination sont estompées, la divination étant une pratique magique comme les autres. Ensuite, il analyse la complexité des éléments qui configurent ces sortilèges et recettes divinatoires, ainsi que la pratique magique en général : les prêtres et l'activité des temples, les risques d'un entourage hostile, la considération positive de ces prêtres pendant ces siècles en certains milieux, le nouveau profil des " prêtres-lecteurs » et les types de divination pratiqués. Il ajoute quelques remarques à propos des particularités de deux textes complexes, la «Liturgie de Mithra » et la « Kosmopoiia de Leiden».

Abstract: This article discusses several aspects of the divinatory spells included in the Greek magical papyri of Roman Egypt. First, it is argued that in these texts the boundaries between magic and divination are blurred - divination is but one of a variety of magical practices. An analysis then follows of the complexity of elements that shape these divinatory spells and recipes, as well as the practice of magic as a whole: priests and the activity of the temples, the risks of a hostile environment, the positive consideration of these priests throughout the centuries in particular "milieux", the new profile of the "lector-priests", and the types of divination practiced. Some observations are also made concerning the distinctive features of two complex texts, the "Mithras Liturgy" and the "Leiden Kosmopoiia".

\section{Introduction}

Depuis l'époque hellénistique, la vie et la culture des Grecs sont soumises à des tensions multiples. Entre les divers traits qui contribuent à modifier profondément le profil social et culturel de cette époque, je voudrais souligner dans cet article le phénomène de l'interculturalisme ou, si l'on préfère, de la fusion d'éléments culturels. En effet, depuis un certain temps, je me suis engagé dans une recherche qui a pour but de repérer les enjeux du processus de fusion culturelle (pour employer un mot « simple») qui conduit à l'élaboration de ces extraordinaires documents connus comme 'papyrus magiques grecs', auxquels il faut

1 Étude réalisée dans le cadre du Projet de Recherche du MICINN (Espagne), FFI201127438. Je remercie ma collègue et amie Hélène Rufat pour la révision de mon texte français. Je remercie vivement l'anonyme 'referee' d'une première version de ce texte, qui a contribué à une amélioration substantielle des références au monde égyptien, ainsi qu'à l'enrichissement des références bibliographiques. Néanmoins, la responsabilité de l'ensemble et des idées ici exposées est tout à fait mienne. 
ajouter évidemment les papyrus démotiques si on veut avoir un cadre complet des questions à analyser.

À quelques exceptions près, quand on aborde l'étude des papyrus magiques, on perçoit, me semble-t-il, une sorte de déformation de l'angle de vue selon que l'analyse est faite par un helléniste ou par un égyptologue : au risque de simplifier, on constate que ces textes sont considérés soit comme une sorte de dérivation de la magie grecque, soit comme un simple «maquillage» de la magie égyptienne, profitant de l'instrument de diffusion qu'est alors devenue la langue grecque. Or, la situation est bien plus complexe. Je pense qu'il faut encore affiner nos analyses pour détecter les procédés qui ont permis d'arriver à la production de ces documents exceptionnels. C'est donc dans cette perspective et ce but que s'inscrivent les remarques qui suivent.

\section{Divination, magie et religion}

Il est indispensable de commencer par la question terminologique. La catégorisation séparée de magie et divination, comme celle de magie et religion, ne se justifient ni dans la perspective égyptienne ni dans celle des Grecs². D'ailleurs, les pratiques religieuses des Égyptiens présentent des aspects qui, du point de vue des Grecs, sont complètement étrangères à leur 'commerce' avec le divin et, par conséquent, peuvent être considérées, pour le moins, comme marginales et à la limite de ce que notre terminologie moderne désigne comme magie (ou même, pour un Grec, comme impiété). Or, il faut partir de l'importance, dans la religion égyptienne, d'un terme (personnifié dans l'iconographie comme une divinité) qui embrasse des aspects qui effacent la dichotomie religion/magie : heka. Tous les dieux et les êtres surnaturels, ainsi que les rois et, dans une certaine mesure, les morts, sont censés le posséder, de la même façon que les divinités, les astres et les morts en état de béatitude détenaient une autre force «magique », appelée akhou ${ }^{3}$. L'emploi de ces forces était nécessaire pour la préservation de la vie, et de son usage correct dépendait l'existence du monde et même des dieux. Maintes cérémonies religieuses, ordinaires et extraordinaires, comportaient des éléments que les modernes auraient appelés «magiques ». Les traités médicaux présentent un mélange de pratiques plus techniques avec des recommandations susceptibles d'être étiquetées comme magiques. L'usage de pratiques magiques était plus ou

\footnotetext{
${ }^{2}$ La polémique est ancienne; cf. déjà BOuCHÉ-LECLERCQ (2003²), p. 32-35, qui défend la séparation, mais en acceptant des points de contact. À l'extrême opposé, par exemple, RiTNER (20084), p. 4-35 (mais il s'agit surtout d'une analyse du beka égyptien et du rôle du dieu Heka). Pour un état récent de la question, cf. JOHNSTON (2008), p. 21-30 et 175-179.

${ }^{3}$ Sur les nuances qui permettent de différencier ces deux termes (beka et akbou), je renvoie aux conclusions faites par KoENIG à la fin du volume des Actes du Colloque du Louvre; cf. par exemple : «Le dieu crée grâce au pouvoir magique (akhou) qui émane de lui. Le corps des dieux est 'plein de magie' (hekaou)", comme nous le disent les Textes des Pyramides » (KoENiG [2002], p. 409).
} 
moins répandu, mais c'est surtout la pratique institutionnelle qui a donné comme résultat que les temples, et plus concrètement la partie connue comme 'Maison de Vie' (Per Âkh), soient devenus les centres où la tradition de savoir 'magique', et de sagesse en général ${ }^{4}$, s'est conservée tout au long des siècles et que ces connaissances soient devenues une partie substantielle du bagage intellectuel des prêtres,

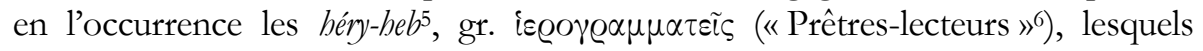
seront à l'époque impériale les principaux responsables de la diffusion des pratiques magiques telles que nous les trouvons dans les papyrus, par un processus « d'adaptation au milieu » qui affecte autant les prêtres que la nature des textes et des pratiques ${ }^{7}$.

Il ne s'agit pas de produire un résumé des traits de la magie égyptienne et de les mettre en contraste avec la magie des Grecs et des autres peuples de l'Antiquité, mais plutôt de se concentrer sur la divination ${ }^{8}$. Le terme est en fait très large et doit être délimité pour cette enquête. La mantique embrasse non seulement la «curiosité » et l'intérêt pour l'avenir (d'une personne, d'un groupe, d'une ville), mais aussi (et surtout), c'est un recours pour résoudre une crise (également personnelle ou collective), en cherchant la réponse divine et/ou la révélation qui mette fin à l'inquiétude face à l'inconnu. Dans le monde ancien, la divination joue un rôle fondamental, que ce soit dans l'espace publique ou dans la vie privée. Elle présuppose la croyance en la possibilité de communiquer avec le divin par des moyens diversifiés, mais qui recourent, presque sans exception, à des personnes qui sont censées avoir des qualités susceptibles de leur permettre d'établir cette communication (volontairement ou involontairement), ou qui sont en possession des moyens techniques leur ouvrant la voie à la connaissance de l'inconnu. À cette distinction, qui rappelle intentionnellement la dichotomie de

4 ASSMANN (2003), p. 73 : «The House of Life was the center of cultural endeavor to preserve and ensure the ongoing progress of cosmic, political, and social life. »

${ }^{5} \mathrm{La}$ transcription de ce terme dans le domaine anglo-saxon est normalement $h r y$ - $h b$, mais il faut constater une évolution phonétique historiquement variable et des formes abrégées, analysées par Quagebeur (1987).

${ }^{6}$ En réalité, comme le signale KoENIG (1994), p. 20-21, cette désignation correspond en principe à un simple lettré, avec une fonction ritualiste ("porteur du rouleau »), qui peut aussi atteindre une position honorifique, en tant que "prêtre lecteur en chef » (khery-heb hery-tep). En tout cas, ils sont mis en rapport avec les opérations magiques étant donné que « la magie était pour les Égyptiens essentiellement une science livresque et la connaissance, ou au moins l'accès à ces recueils magiques, donnait au magicien toute sa puissance » (KoENIG [1994], p. 20).

${ }^{7}$ Fondamentaux à ce propos les travaux de FrANKFurTER $(1997,1998,2005)$.

8 Très remarquable pour la fonction de la divination dans la magie est ce que nous lisons dans

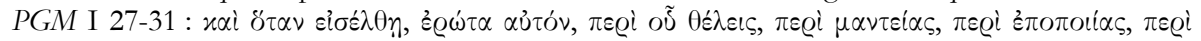

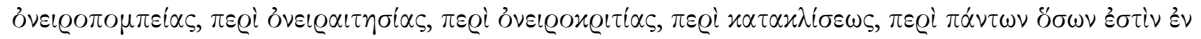
$\tau \tilde{\eta} \mu \alpha \gamma \iota \tilde{\eta} \dot{\varepsilon} \mu \pi \varepsilon \iota \rho^{\prime} \alpha$, « et, quand il entre, interroge-le sur tout ce que tu veux, sur la divination, sur la composition en vers, sur l'envoi de songes, sur la demande de songes, sur leur interprétation, sur la période de repos, sur tout ce qui est le sujet des pratiques magiques ». Une belle transformation d'Apollon en maitre magicien, qui nous offre un catalogue des pratiques divinatoires et nous confirme le caractère central de la divination dans les pratiques magiques. 
Cicéron, s'en ajoute une autre, entre la divination pratiquée dans les sanctuaires et la divination individuelle ${ }^{9}$. En outre, les réponses divines et/ou les oracles émis par les intermédiaires de la divinité peuvent être réunis par écrit et devenir un instrument polyvalent, tant du point de vue religieux que politique : entre parole et écriture la réponse oraculaire est véhiculée avec une multiplicité d'enjeux. Finalement, et pour ne pas trop élargir cette énumération de traits généraux, on doit souligner l'importance, dans les sociétés anciennes, des professionnels de la divination, que ce soit comme personnel des temples ou des cours royales, ou en tant que divins réputés entourés d'un grand prestige, parfois avec une lignée de famille consolidée ${ }^{10}$.

\section{Les textes des PGM: le miroir d'un monde complexe}

Ces textes reflètent bien la complexité du monde qui les entoure, du point de vue de la culture et des croyances. C'est un monde profondément marqué par le mélange de traditions culturelles à tous les niveaux. Des différents aspects qu'on pourrait identifier, je vais en choisir quelques-uns que je considère comme représentatifs, afin de mieux comprendre le processus de « transculturalité » qui se dégage de ces documents ${ }^{11}$.

\subsection{Contextes, fonction, acteurs}

La divination en Égypte a des racines anciennes, à en juger par le témoignage des différents textes de toutes les périodes, depuis l'Ancien Empire jusqu'à l'époque hellénistique. L'information qui s'en dégage nous permet de repérer des points de contact et de divergence sous trois aspects : les contextes de la divination, ses acteurs et les modalités de la consultation mantique ${ }^{12}$.

9 En fait, celle-ci s'adapte de façon plus exacte à la réalité de la pratique divinatoire. JOHNSTON (2008), p. 9, considère raisonnablement que la classification cicéronienne est « more heuristic than real ». Cf., dans le même sens, SuÁrEZ DE LA TORRE (2005), p. 29-31.

${ }^{10}$ Cf. infra. Les traits principaux des prêtres et de leurs fonctions ont été clairement identifiés par SAUNERON (1998).

${ }^{11}$ Un appel à la prudence au moment d'analyser ces textes se justifie par la grande variété qui se cache sous la dénomination de "papyrus magiques» (autant grecs que démotiques). Chaque recette ou «spell» a une histoire ou une généalogie différente et, en plus, la compilation finale donne à chaque « grimoire » une nature différente.

12 À quelques exceptions près (par exemple LECLANT [1968]) les études sur la divination en Égypte abordent des aspects concrets. Vid. SAUnERON (1959), DunAND (1997), QuAEGEBEuR (1997), Demichelis (2002), Ritner (2002a, 2002b). Néanmoins, il faut tenir compte de deux faits : (a) les références à la divination sont fréquentes dans les études sur la magie en Égypte en général (citées dans cet article) et (b) «... la divination dans la civilisation égyptienne relève de la magie au sens où elle se fonde sur la même relation signifiant-signifié, alors que du point de vue égyptien ce n'est pas de la magie : elle ne vise pas fondamentalement à un agir mais à un savoir » (KoENIG [2002], p. 412, n. 6). 


\section{L'activité des temples}

L'évolution de la divination en Égypte est indissociable du fonctionnement des temples et de leur concentration absolue de l'activité religieuse. Il n'y a pas en principe un dieu qui préside à l'activité divinatoire, à l'exception de l'existence ancienne de centres spécifiquement oraculaires, comme celui d'Amon, et de certains développements tardifs, comme la spécialisation oniro-médicale du temple d'Imhotep à Memphis ou l'évolution oraculaire du dieu Bès ${ }^{13}$. En plus, la divination égyptienne est contrôlée par les prêtres et connaît en principe une orientation plutôt officielle et très souvent 'politique'. Les réponses connues ont généralement comme destinataires le pharaon, sa famille et leurs fonctionnaires de haut rang. Très fréquemment, les oracles traitent aussi des questions relatives aux prêtres et à leurs familles. Les temples sont le centre habituel où se réalisent les opérations connues comme $p H-n T r$, lit. «accéder à un dieu », qui n'est qu'un rite de révélation, cherchant normalement la réponse d'une statue divine ${ }^{14}$. Néanmoins, les consultations privées des oracles, d'une part, et, de l'autre, le recours à des moyens non strictement 'officiels' pour avoir des réponses aux inquiétudes les plus diverses revêtent une importance de plus en plus grande. Même pendant la période pharaonique, les héry-beb ne se bornaient pas à travailler en exclusivité pour le temple, renfermés dans la Maison de Vie : ils consacraient une partie de leur temps à des affaires privées ${ }^{15}$. La " privatisation » de la consultation oraculaire est un phénomène progressif, caractérisé par l'apparition de formes alternatives de divination en dehors de la consultation prophétique plus formelle. Les $P G M$ confirment l'importance acquise par le recours privé à la divination, même si les prêtres essaient de maintenir parfois la solennité formelle des consultations plus institutionnelles. D'ailleurs, les formes de consultation mantique à l'époque tardive se multiplient et on enregistre une remarquable série de «mutations $»^{16}$, ce qui n'empêche pas la naissance de nouveaux centres oraculaires dédiés aux divinités les plus importantes de cette période. La divination privée est assumée par les prêtres; les traditions oraculaires « des temples » se mêlent aux méthodes et pratiques privées. Or, les $P G M$ nous révèlent l'effort des héry-heb pour monopoliser ce qu'était la magie (sous ses deux aspects), à cette époque-là, mais qu'ils pratiquent comme simple démonstration du vieil he-ka, y compris la connaissance de ce qui est occulte, c'est-à-dire la divination. Le bierogrammateus se recycle; la divination (comme la magie) est une «commodity $»^{17}$, mêlée à d'autres exigences des clients. D'ailleurs, il faut souligner que les prati-

13 DUNAND (1997).

${ }^{14}$ RitNeR (2008)), p. 214-220.

15 RitNER $\left(2008^{4}\right)$, p. 232 : «Combining in himself the roles of composer, compiler, and performer, it is the priest alone who constitutes the "private" magician in ancient Egypt. »

${ }^{16}$ FRANKFURTER (1997).

${ }^{17}$ MOYer (2003), p. 72. 
ques divinatoires des Égyptiens et des Grecs n'étaient pas trop différentes ${ }^{18}$. En tout cas, comme on le verra par la suite, la variété de pratiques divinatoires enregistrées dans les papyrus magiques est relativement limitée.

\section{Courir des risques}

Il serait très risqué de laisser de côté le contexte historique plus immédiat en ce qui concerne la situation des pratiques magiques et divinatoires dans l'Empire Romain pendant cette période. Les interdictions contre ces pratiques (mais surtout contre la consultation oraculaire) se répètent sous le gouvernement de presque tous les empereurs ${ }^{19}$. La très célèbre expression sileat omnibus perpetuo divinandi curiositas de l'édit de 359 de Constance II $^{20}$ contre les consultations de toutes les espèces possibles de malefici (en l'occurrence, haruspex, mathematicus, hariolus, augur, vates, chaldaeus, magus) connaît un remarquable précédent dans le décret du préfet d'Égypte Q. Aemilius Saturninus en 198-9 (sous le mandat de Septime Sévère), contre ceux qui se « laissent tromper par de nombreuses sortes de divination ». Il déclare l'interdiction « de cette curiosité dangereuse » (lit. "glissante», «trom-

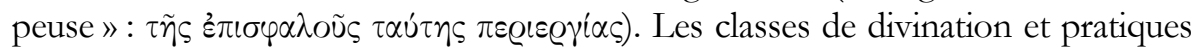
proscrites expressis verbis sont : les oracles, les écrits censés avoir été donnés par les dieux et la prétention d'avoir une connaissance des choses supérieure à celle du reste des mortels grâce à des pratiques comme la "procession de statues» $\left(\varkappa \omega \mu \alpha \sigma^{\prime} \alpha\right)$ et d'autres sortes semblables de $\mu \alpha \gamma \gamma \alpha \nu \varepsilon^{\prime} \alpha^{21}$. Cette politique d'interdiction sera évidemment inutile, mais elle nous permet de comprendre le climat qui entourait parfois la pratique de la consultation divinatoire et le fait que la diffusion de textes tels que les PGM avait une valeur de "résistance » culturelle contre les Romains. Je dis «parfois", parce que l'attraction pour l'activité des " prêtres » égyptiens ne fut pas toujours considérée négativement, à en juger par le succès de quelques-uns de ces personnages, comme on va le voir.

En outre, du point de vue de la méthode, s’interroger sur la divination indépendamment de l'ensemble des opérations magiques n'est pas complètement justifié. Il y a certes des documents dans cet ensemble qui sont purement « divinatoires » (sortes, quelques oracles chrétiens, homeromanteion), mais les papyrus à contenu magique proprement dits présentent le traitement de la divination comme un résultat, entre autres, des opérations magiques, dans la lignée des autres avantages résultant de l'efficacité de ces opérations. Très fréquemment, les éléments mantiques sont mis en série avec d'autres composants de l'activité magique : par exemple, une phialomancie aspire surtout à la possibilité de disposer de l'aide et de la révélation d'un dieu pour des finalités diverses (cf. infra). Le

\footnotetext{
18 À quelques exceptions près, naturellement, comme les mouvements prédictifs de la barque d'Osiris (vid. SAUNERON [1998], p. 113-115).

${ }^{19}$ Cf. RitNer (20084), p. 217-220.

${ }^{20}$ CTh 9, 16, 4. Pour l'histoire de ces prescriptions vid. DESANTI (1990); sur ce texte, p. 147.

${ }^{21}$ Pap. Yale inv. 299 (Coll. Youtie I); édité par PARÀssoglou (1976); cf. LEWIS (1977).
} 
décret commenté plus haut montre que, même si les Romains craignaient surtout l'effet des oracles, ils contemplaient cette activité comme une partie de ce qui est décrit péjorativement comme $\mu \alpha \gamma \gamma \alpha \nu \varepsilon i \alpha^{22}$.

\section{L'entourage positif}

Les $P G M$ forment un ensemble très représentatif d'une période historique qui connaît, en matière religieuse, des phénomènes changeants et des interactions parfois surprenantes (dans le cadre complexe qui se cache sous le terme de "syncrétisme »). Dans un monde où des courants religieux proclament le besoin de l'unité avec le divin et les possibilités de révélation divine par plusieurs moyens, où la philosophie s'entremêle à des traditions diverses et devient théosophie, où l'on croit à la possibilité d'animer les statues, où existent des êtres extraordinaires, des theioi andres, qui ressuscitent les morts et font des miracles, où un philosophe doit se défendre d'une accusation de pratiques magiques pernicieuses en démontrant qu'il connaît bien ces pratiques, et où les poètes se servent de sorcières (une vieille tradition) qui font prophétiser les morts, dans un tel monde, les textes des papyrus magiques ne sont pas un phénomène isolé, mais un exemple extraordinaire d'une autre koiné, qui n'est pas linguistique, mais culturelle et religieuse, avec une nouveauté : elle est la création des Égyptiens « hellénisés », c'est, enfin, le fruit d'un milieu très particulier. C'est aussi le miroir où les prêtres projettent une image telle qu'ils pensaient que les autres voulaient la contempler : ils attiraient le regard de l'autre en montrant ce qu'ils pensaient que les autres voulaient regarder, mais en s'efforçant de garder le profil de leur altérité23.

\section{Les acteurs : remarques sur les « prêtres-lecteurs »}

\section{Les 'anciens' héry-heb et la tradition prophétique en Égypte 24}

Les témoignages sur les prêtres-lecteurs sont assez abondants ${ }^{25}$. Un exemple souvent cité est constitué par les récits qui se trouvent dans le Papyrus Westcar, un

\footnotetext{
22 Cf. Platon, Leg. 908d, 933a.

${ }^{23}$ Je suis conscient que la question de l'évolution des prêtres et de leurs fonctions pendant la période impériale est loin d'être simple. Pour commencer, le terme 'prêtre' désigne historiquement un ensemble dont les fonctions sont très diversifiées et parfaitement définies en relation au fonctionnement du temple et des différents rites (cf. SAUNERON [1998], p. 39-87 et passim, et DieLEMAN [2005], p. 205-220). Mais il faut tenir compte du fait que l'évolution du rôle des prêtres et surtout leur considération sociale est liée aux circonstances et conditionnements de la religion égyptienne au long des siècles de domination romaine. Dans ce cadre évolutif on enregistre un besoin de la part du clergé de s'adapter aux nouvelles circonstances, ce qui conduit à un raffinement des tactiques pour maintenir un status reconnu par la société, qui se produit de façon parallèle à la nouvelle orientation, plus locale et proche aux besoins pratiques, des temples. Cette adaptation aux nouvelles circonstances est spécialement visible dans le cas du scriptorium et, en général, de la Maison de Vie. Les prêtres-lecteurs auront un rôle décisif dans la tâche de maintenir en équilibre entre tradition et innovation, ou, dans les termes employés par FRANKFURTER (1998), p. 241, "preservation and syncretism ». Cf. en tout cas infra, n. 29.

${ }^{24}$ Un traitement plus approfondi de la présence des prêtres dans «l'imaginaire » littéraire égyptien et chez les auteurs grecs et latins se trouve dans DiELEMAN (2005), p. 221-254.
} 
ensemble de contes du type des Mille et une Nuits. Cette fois ce sont les fils du pharaon Chéops (IVe dynastie) qui racontent des histoires à leur père pour faire partir son ennui. Dans les contes de la partie conservée le rôle décisif est joué par des prêtres-lecteurs et par un ancien doué de pouvoirs extraordinaires : les deux premiers sont capables, respectivement, de donner la vie à un crocodile qui dévore l'amant de la femme infidèle ou de séparer en deux et superposer les eaux d'un fleuve pour récupérer un bijou. L'ancien Djédi a le pouvoir de faire revivre un homme ou animal auquel on aurait fait couper la tête et fait une prédiction sur la dynastie qui régnera en Égypte. Ce pouvoir de prédiction était aussi un trait des prêtres-lecteurs. On peut l'illustrer avec le récit du Pap.Pet. 1116B et le lecteur Néferty qui raconte au pharaon Snéfrou (à sa demande) une longue prophétie aux traits apocalyptiques avec le motif du « roi sauveur qui viendra de l'Orient » dont le texte primitif pouvait remonter à l'an 2000 av. J.-C., ce qui nous donne un exemple de l'extraordinaire antiquité d'une typologie prophétique qui deviendra abondante pendant les époques hellénistique et romaine ${ }^{26}$.

\section{Les nouveaux hierogrammateis : continuité et renouvellement}

La capacité d'adaptation et de renouvellement des prêtres-lecteurs a été très remarquable, grâce a leur formidable formation et leur habileté pour maintenir leur status. Ceux qui, devenus magiciens, ont laissé leurs recettes dans les PGM présentent un profil qui illustre très bien les circonstances historiques et sociales des siècles de la domination romaine. Ils sont pleinement ouverts à l'assimilation des éléments qui proviennent des cultures voisines et ils ont parfaitement adapté cet ensemble documentaire aux besoins les plus divers. Ils ne constituent pas une classe isolée dans la «Maison de Vie » et éloignée du monde. Le mouvement se produit dans les deux sens : les gens sont à la recherche des 'magiciens' et ceux-ci sont à la recherche du contact avec les centres de pouvoir et de culture ${ }^{27}$.

Au-delà des possibilités de réalité historique concrète, nous avons un exemple bien connu de la célébrité des magiciens égyptiens en dehors des frontières de

25 Je renvoie à nouveau aux ouvrages de SAUNERON (1998), FrANKFURTER (1998), RitNER $\left(2008^{4}\right)$, ainsi que PINCH $\left(2009^{2}\right)$. Les textes égyptiens qui seront cités ici ont été traduits en français et commentés par LEFEBVRE (1948) ou GRANDET (1998).

${ }^{26}$ Je renvoie à l'Introduction de SUÁREZ DE LA TORRE $\left(2002^{2}\right)$.

${ }^{27}$ Je ne soutiens pas pour autant que les formulaires magiques des papyrus ont été composés dans leur totalité par les prêtres-lecteurs. Parfois, l'origine grecque est plus qu'évidente, surtout dans les papyrus de datation plus ancienne : pensons au sortilège de Philinna contre la migraine (PGM XX, 1319). Mais la plupart de ces textes sont nés dans un milieu égyptien, ont été transmis et copiés par des Égyptiens hellénisés et finalement arrangés par des copistes des scriptoria égyptiens. Pensons que la plus grande collection de textes magiques de PDF provient d'un seul lieu : c'est l'ensemble connu comme 'bibliothèque Anastasi', d'origine Thébaine. Il serait long d'énumérer la série des papyrus qui enregistrent un contenu où l'on détecte la main des prêtres-lecteurs devenus magiciens, mais il faut également tenir compte du fait que parfois on perçoit des auteurs hors du cercle sacerdotal du temple, comme l'a démontré DIELEMAN (2005), p. 185-203, même si le 'narrateur' fait constamment référence à leur autorité magico-religieuse. D'ailleurs, rappelons que la diffusion des « livres magiques » et de ses pratiques était due aussi aux magiciens itinérants (voir DICKIE [2001], p. 221-242). 
l'Égypte. Le personnage nommé Thessalos, protagoniste du récit pseudoépigraphe qui précède l'ouvrage De virtutibus herbarum, va à la recherche d'un vrai spécialiste qui puisse le faire arriver à la contemplation directe d'un dieu (le $p h \underline{h}-n \underline{t} r$ égyptien, traduit en $\sigma u ́ \sigma \tau \alpha \sigma \iota \varsigma$ dans nos papyrus) ${ }^{28}$. Mais nous avons des exemples non moins célèbres du caractère parfois itinérant de ces prêtres. Rappelons le Zatchlas de la Métamorphose d'Apulée, mentionné dans le récit de Téliphron chez Birrena $^{29}$, qui ressuscite temporairement un cadavre. Les deux aspects (activité en Égypte et ailleurs) coïncident apparemment dans le Philopseudês de Lucien. Eucratès affirme avoir établi une relation avec Pancratès, maître d'Arignotos, et être devenu pour un court délai un catastrophique "apprenti sorcier». Ce Pancrates, «qui ne parlait pas purement la langue grecque $»^{30}$ et qui était doué de pouvoirs extraordinaires, parfaitement en correspondance avec ce que nous lisons dans les textes des papyrus ${ }^{31}$, pourrait être ${ }^{32}$ un personnage historique, du temps d'Hadrien, et en tout cas sa proximité avec le Pachrates du PGM IV 33 est plus que surprenante. Quoi qu'il en soit, la présence de cette catégorie de prêtres dans la vie publique de l'Empire ne fait aucun doute, comme le montre le cas du prêtrephilosophe (stoïcien) d'Alexandrie Chaerémon ${ }^{34}$, qui nous donne un exemple d'intellectuel qui ne s'adapte pas précisément au profil qu'il nous présente de la vie contemplative des plus anciens prêtres égyptiens ${ }^{35}$ (encore moins si l'on accepte son identification avec le précepteur homonyme de Néron) ${ }^{36}$.

${ }^{28}$ SMith (1978), p. 172-189; RitNer (1995), p. 335-338; MoYer (2003); (2011), p. 208-273; SFAMENI GASPARRO (2010).

${ }^{29}$ Met. III, 21-30: Zatchlas intervient au ch. 28.

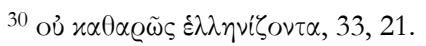

${ }^{31}$ Mise à part la description de la scène de transformation des outils domestiques en servi-

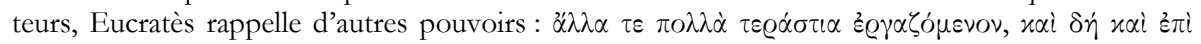

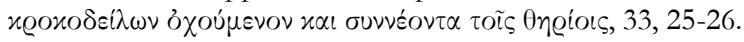

32 Cf. Ogden (2007).

33 2441-2621 et 2446-2455.

${ }^{34}$ Sur ce personnage on peut consulter l'introduction de VAN DER HORST (1984).

${ }^{35}$ Le fr. 10 Van der Horst (= Porph., De abst. IV, 6-8) nous offre une intéressante classification des fonctions des serviteurs des temples égyptiens avec des remarques sur leur formation plus ou moins philosophiques. Dans le groupe des mieux doués pour la philosophie se trouvent

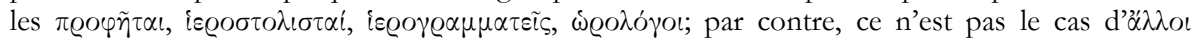

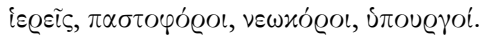

${ }^{36}$ En outre, les textes $P G M$ et d'autres récits littéraires grecs nous donnent un profil assez exact de la conception qu'on avait de ces mages-divins : cf. par exemple, PGM I, 42-195, avec une « initiation» (BETZ [1990], p. 209-229, MOYER [2003]) didactique sous forme de lettre (de Pnouthis à son disciple Kêryx), ainsi que le premier des récits de Setne Khaemwaset et l'histoire du livre de Thot déposé dans le tombeau de Naneferkaptah (Pap. Caïre 30646. Première publication : GrifFith [1900]. Cf. LichtHeIM [2006²], vol. III, p. 126-138, avec la bibliographie; voir maintenant AGUTLABORDÈRE - CHAUVEAU [2011]). Le personnage historique qui a donné lieu à ce récit est le prince Khaemwaset, quatrième fils de Ramses II (ca. 1184-1153), très connu par ses intérêts «antiquariens ». Le cycle des légendes sur Setne-Khamwas appartient à une datation grosso modo entre le $\mathrm{II}^{\mathrm{e}}$ siècle av. J.-C. et le II e ap. J.-C. Voir les remarques de RitNeR (20084), p. 63-64, à propos de 


\section{Les modalités}

La divination dans les textes magiques est sans doute indissociable du reste des pratiques magiques ${ }^{37}$ et elle présente la même fusion d'éléments interculturels, mais, à mon avis, avec un poids très fort du canevas égyptien qui donne un support aux diverses innovations ou aux concessions aux traditions grecques et d'origines diverses ${ }^{38}$. On peut classifier les pratiques 'divinatoires' qu'on repère dans les PGM de la façon suivante : 1. Communication « directe avec les dieux et les démons ». Celle-ci est susceptible d'être divisée en : (a) vision directe ou bien provoqué grâce à un rite, soit en état de veille soit en dormant, (b) demande de l'envoi d'un rêve divinatoire et (c) une épiphanie provoquée par des moyens 'techniques' (lychnomancie, lécanomancie ou phyalomancie); 2. Communication moyennant des intermédiaires (le rôle du 'medium'); 3. Nécromancie ${ }^{39}$. De cet ensemble je voudrais souligner le poids de la tradition égyptienne et de sa vision des rapports entre les hommes et les dieux, entre les vivants et les morts, entre l'homme et la nature. Autant du point de vue général que dans leurs détails précis, on part d'un schéma que, en conservant des traits anciens, continue en vigueur dans l'entourage Égyptien contemporain, comme l'on peut vérifier grâce aux textes démotiques incorporés aux $P G M^{40}$. Mais il faut remarquer aussi que, par coïncidence typologique ou par la tendance historique à l'assimilation des pratiques, en raison de contacts culturels et, surtout, de la forte influence grecque pendant la période hellénistique, l'ensemble ne s'éloigne pas trop des pratiques

l'importance de ce texte pour comprendre la valeur sémantique de $p h r$ en tant que «encercler» comme synonyme de « charmer».

37 À cet égard, il est très significatif que l'œuvre de PINCH (2009²) ne contienne aucun chapitre spécifique sur la divination (et pour cause). Je renvoie à cet ouvrage pour les nombreuses références aux modalités magiques à caractère divinatoire. D'autre part, dans l'ensemble des papyrus magiques, il faut tenir compte du fait que le PDM XIV, même s'il est difficile d'établir le niveau d'influence (lire : «traduction ») des textes grecs sur cet exemplaire démotique, serait représentatif de la tradition magique égyptienne. Or, la majorité des sortilèges qu'il contient (95) appartient à une de ces modalités : médicale (25), divinatoire (23) et érotique (19, y compris les sortilèges de « rupture »).

${ }^{38}$ Un résumé des pratiques divinatoires égyptiennes se trouve dans l'article de LECLANT (1968). Il réunit d'abord une liste dans un chapitre sur 'Oracles et prophéties '; puis viennent les 'méthodes employées': Mouvement de la barque divine, questions posées par ostraca, oracles rendus par le dieu ou 'décrets' (parfois sur l'au-delà), statues parlantes, comportement des animaux sacrés, vaticination des possédés. Quant aux «domaines d'application des oracles et prophéties » il signale leur rôle important dans la haute politique, mais avec le nouvel Empire l'oracle d'Ammon s'occupe aussi des affaires privées. Autres pratiques: Oniromancie, incubation, omen, ordalies (tendance des oracles entre 1085-950), prophéties des moribonds (cf. l'agneau du roi Bocchoris), prophétisme, astrologie. Aucun mot sur la nécromancie, pour laquelle voir RITNER (2002). Pour des analyses plus spécifiques, généralement en rapport avec les papyrus magiques grecs, vid. HOPFNER (1921-1924), passim, ESCHWEILER (1994), EItrem (1997), Gordon (1997), Dieleman (2003), FrankfurTER (2005), SZPAKOWSKA (2003a, 2003b, 2006).

39 SuÁreZ DE LA TORRE (2009b). On peut comparer avec la classification de JOHNSTON (2008) : «Seeing it yourself, Fire and Water, Sending Dreams, Receiving Dreams, Divinatory Statues, Mills and Spheres, Skulls and Corpses ». La séquence typologique est à peu près la même, car la rubrique « Divinatory Statues » ne fait pas référence, précisément, aux PGM.

${ }^{40}$ Je renvoie aux analyses et données des œuvres citées supra, n. 37-38. 
helléniques et que les auteurs compilateurs offrent un résultat qui pourrait être qualifié de koiné mantique.

Parmi les éléments où le poids de la tradition égyptienne est le plus remarquable, on trouve ${ }^{41}$ :

(a) La façon de se diriger aux dieux dans les logoi des opérations magiques. Il est très important de constater que, bien que cette divinité puisse être bien identifiée, dans le même texte on aperçoit des oscillations, en consonance avec la tendance à établir parfois des séries de divinités qui embrassent des aspects semblables (par exemple les divinités solaires).

(b) L'importance des visions oniriques, qui n'est qu'une partie du rôle que les songes jouent dans maints rituels et qui est très cohérente avec la longue tradition de pratique d'incubation médicale ou révélatrice en général. C'est aussi une pratique bien facilement assimilable aux pratiques helléniques.

(c) Le rôle du monde d'outre-tombe, ou plus exactement du Douat, et les traditions sur la possibilité de communication avec les morts, l'immortalisation par immersion dans le Nil et, encore plus, la familiarité avec la manipulation des cadavres, nous donne une moyenne remarquable de pratiques où les morts sont présents.

(d) Le recours à une pharmacologie magique de longue tradition.

(e) L'existence d'une longue tradition hymnique.

(f) La fabrication d'objets : statues, anneaux, gemmes, phylactères.

(g) L'emploi du médium.

Entre les éléments plus purement grecs, en plus de la majorité de ceux qui viennent d'être indiqués, on peut souligner :

(a) Le poids de certaines traditions mythiques et/ou littéraires (surtout autour d'Apollon).

(b) Le développement des possibilités d'établissement de liens à travers les traits de certaines divinités, comme, par exemple, Hermès.

\subsection{Deux cas particuliers : la « Liturgie de Mithra » et la « Kosmo- poiia de Leiden "}

En outre, il faut tenir compte des nouveaux développements spirituels résultant de la naissance de nouveaux courants. En effet, ces textes constituent un ensemble d'une variété immense : des formulaires très synthétiques et pratiques à côté de quelques parties plus complexes, qui sont le fruit de la complexité des croyances qui s'enregistre depuis l'époque hellénistique. Un exemple remarquable

${ }^{41}$ Cf. ci-dessus, n. 30 et 31. 
est le long sortilège connu comme Liturgie de Mithra ${ }^{42}$. C'est un texte extraordinaire, un mélange exceptionnel d'éléments divers autant dans la forme que dans

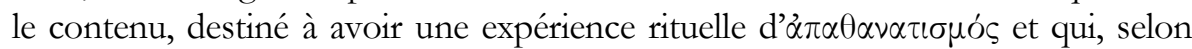
une définition de Betz, nous permet d'apercevoir le commencement de l'hermétisme, mais sans être encore chargé des forts composants gnostiques ultérieurs ${ }^{43}$. En ce qui concerne la divination, insistons sur le fait que, précisément au moment central de la contemplation de Mithra, et lorsque le pratiquant du rite vient d'expérimenter son « immortalisation » et sa « renaissance », le texte accumule des instructions détaillées pour demander au dieu une révélation oraculaire, accompagnée de trois possibilités alternatives de la mener à terme (718-743) ${ }^{44}$. Au point qu'on pourrait penser que, en réalité, l'expérience complexe de cette recette a pour but une consultation oraculaire directe : c'est-à-dire qu'il s'agit d'une variété du $p H-n T r$, déguisée en expérience mystique et mystérique, mieux adaptée aux courants de ce temps.

Non moins fascinant et complexe est le contenu du PGM XIII, avec deux versions $^{45}$ d'un rite divinatoire et, apparemment, partiellement initiatique, qui contient comme texte central de la cérémonie, une théogonie-cosmogonie qui mêle des traits égyptiens anciens à d'autres éléments d'origine diverse et plus récents ${ }^{46}$. Sans entrer dans la question des caractéristiques formelles de ces versions et de la formation du papyrus, il faut remarquer que, comme dans le cas de la pseudo-liturgie mithriaque, le but essentiel de la pratique centrale et des prières qui la composent n'est que celui d'obtenir du dieu suprême une prédiction sur le destin qui est réservé au consultant à partir de la date de naissance et de la position des astres. En effet, les deux textes du papyrus, présentés comme l'œuvre de Moïse ${ }^{47}$, foisonnent d'éléments zodiacaux et de références aux dieux des heures et des semaines. On se trouve, donc, devant une variété astrologique de la consultation divinatoire, en provoquant encore une fois une systasis avec la divinité.

\section{Conclusions}

1. La divination dans les papyrus magiques de l'époque impériale romaine est conçue comme une pratique magique comme les autres.

42 PGM IV, 475-834. Dieterich (1907), Merkelbach (1992), BetZ (2003), Zago (2010), SFAMENI GASPARRO (2011).

${ }^{43}$ BETZ (2003), p. 37-38.

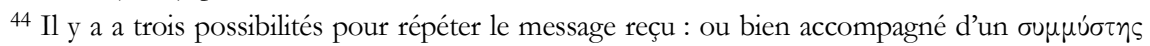
ou en solitaire ou avec un autre, mais en vérifiant préalablement qu'il est en condition de l'écouter.

45 PGM XIII, 1-441 et 442-1078. Il s'agit du Pap. Leid. J 395.

${ }^{46}$ Les études les plus complètes sur cette cérémonie magique sont celles de DieTERICH (1891) et MERKELbACH (1992). Voir maintenant SuÁREZ DE LA TORRE (2013b).

${ }^{47}$ Cf. SuÁreZ de LA Torre (2012). 
2. Les procédés divinatoires de ces textes montrent un haut degré d'harmonisation entre les pratiques égyptiennes et les pratiques grecques.

3. Ces caractéristiques relèvent surtout du fait que les auteurs/compilateurs des textes magiques appartiennent majoritairement (mais pas exclusivement) à la classe des hierogrammateîs, un groupe de prêtres qui, en plus de leur connaissance des textes égyptiens, montrent à cette époque une bonne connaissance d'autres sources, autant authentiques que pseudépigraphiques.

4. La typologie de ces pratiques est légèrement limitée (par rapport à la richesse de méthodes connues dans le Monde Ancien), avec un poids majeur de deux catégories: (a) la consultation divinatoire faisant partie de l'apparition d'un dieu ou d'un démon, produite comme conséquence d'une invocation, accompagnée ou non de manipulations instrumentales; et (b) l'oniromancie (qui peut être fusionnée partiellement avec la catégorie précédente).

5. Le résultat présente une grande diversité, en rapport avec la genèse complexe de chaque papyrus. Entre Apollon et Bès, en passant par Pschai-Aion ou Mithra, avec le concours d'un Hermès-Thot "maître de divination ", et à l'aide de pratiques complexes (et coûteuses) de manipulation des substances les plus exotiques ou anomales, ces papyrus nous donnent un bon éventail des possibilités d'ajouter aux divers effets de la magie une réponse aux problèmes (simples et complexes) posés par la vie quotidienne ${ }^{48}$.

Emilio SUÁREZ DE LA TORRE

Universitat Pompeu Fabra, Barcelona

Departament d'Humanitats

Courriel:emilio.suarez@upf.edu

\section{Bibliographie}

Agut-Labordère, D., Chauveau, M., Héros, magiciens et sages oubliés de l'Egypte ancienne. Une anthologie de la littérature en égyptien démotique, Paris, Les Belles Lettres (La Roue à Livres), 2011.

Assmann, J., Weisheit und Mysterium: Das Bild der Griechen von Ägypten, München, 2000.

-, The Mind of Egypt: History and Meaning in the Time of the Pharaobs, Harvard University Press, 2003.

BETZ, H.D., Hellenismus und Urchristentum (Gesammelte Aufsätze I), Tübingen, J.C.B. Mohr (Paul Siebeck), 1990.

- (ed.), The Greek Magical Papyri in Translation. Including the Demotic Spells (vol. I: Texts), Chicago, $1992^{2}$.

—, The 'Mithras Liturgy', Tübingen, J.C.B. Mohr (Paul Siebeck), 2003.

BonHÊME, M.-A., "Pouvoir, prédestination et divination dans l'Égypte pharaonique », in E. Smadja, É. GenY (éd.), Pouvoir, divination et prédestination dans le monde antique, 1999, 139-158. Borghouts, J. F., Ancient Egyptian Magical Texts, Leiden, Brill, 1978. (1997).

${ }^{48}$ Une valorisation de ces expériences magiques en tant que telles se trouve dans GORDON 
Bouché-LeclercQ, A., Histoire de la divination dans l'Antiquité, Grenoble, Jérôme Million, 2003 (nouvelle édition avec une préface de Stella GEORGOUDI).

Boylan, P., Thoth or the Hermes of Egypt. A Study of Some Aspects of Theological Thought in Ancient Egypt, London, Oxford University Press, 1922 (reimpr. ed. Kessinger, 2003).

Brashear, W.M., "The Greek Magical Papyri: an Introduction and Survey; Annotated Bibliography (1928-1994)", ANRW II, 18.5 (1995), p. 3380-3684.

Brier, B., Ancient Egyptian Magic, New York, Harper Collins, 1980.

Calvo Martínez, J.L., « Morfología de las prácticas mánticas de la luz. Fotagogia y licnomancia en los $P G M »$, in MONACA (2009), p. 45-78.

Ciraolo, L.J., "Supernatural Assistants in the Greek Magical Papyri », in MireCKI - MeYer (2001), p. 279-295.

Ciraolo, L., Seidel, J. (éd.), Magic and Divination in the Ancient World, Leiden, Brill, 2002.

Daniel, R.W., Maltomini, F., Supplementum Magicum, Opladen, I. 1990, II. 1992.

Demichelis, S., «La divination par l'huile à l'époque ramesside », in KoENIG (2002), p. 149-167.

DeSANTI, L., Sileat omnibus perpetuo divinandi curiositas. Indovini e sanzioni nel diritto romano, Milano, Giuffrè Editore, 1990.

Dickie, M.W., Magic and Magicians in the Greco-Roman World, London/New York, Routledge, 2001.

Dieleman, J., «Stars and the Egyptian Priesthood in the Graeco-Roman Period », in Noegel WALKER - WHEELER (2003), p. 137-154.

Dieleman, J., Priests, Tongues, and Rites. The London-Leiden Magical Manuscripts and Translation in Egyptian Ritual (100-300 CE), Leiden, Brill, 2005.

DiETERICH, A., Abraxas. Studien zur Religionsgeschichte desspätern Altertums, Leipzig, Teubner, 1891.

Dodd, D.B., Faraone, Ch.A., Initiation in Ancient Greek Rituals and Narratives, London/New York, Routledge, 2003.

DunAND, F., «La consultation oraculaire en Égypte tardive. L'oracle de Bès à Abydos », in J.-G. HeINTZ (éd.), Oracles et prophéties dans l'Antiquité, Paris, de Boccard, 1997 (Travaux du Centre de Recherche sur le Proche Orient, 15), p. 65-84.

Eitrem, S., Orakel und Mysterien am Ausgang der Antike, Zürich, Rhein-Verlag, 1947.

—, «Dreams and Divination in Magical Ritual», in Ch. A. FAraone, D. ObBink (éd.), Magika Hiera. Ancient Greek. Magic and Religion, Oxford/New York, OUP, 1997, p. 175-187.

EschweIler, P., Bildzauber im alten Ägypten, Göttingen, Univ. Freiburg/Vandenhoeck-Rupprecht, 1994.

FAUTH, W., Helios Megistos. Zur synkertistischen Theologie der Spätantike, Leiden, Brill, 1995.

Frankfurter, D., "Ritual Expertise in Roman Egypt and the Problem of the Category 'Magician' », in SCHÄFER - KIPPENBERG (1997), p. 115-135.

-, Religion in Roman Egypt. Assimilation and Resistance, New Jersey, Princeton University Press, 1998.

—, «Voices, Books, and Dreams: The Diversification of Divination Media in Late Antique Egypt », in JOHNSTON -STRUCK (2005), p. 233-254.

Friedrich, H.-V. (éd.), Thessalos von Tralles. Griechisch und Lateinisch, Meisenheim am Glan, Hain, 1968 (Beiträge zur Klassischen Pbilologie, Heft 28).

GeE, J., « Oracle by Image: Coffin Text 103 in Context », in Ciraolo - Seider (2002), p. 83-88.

Gordon, R., «Reporting the Marvellous: Private Divination in the Greek Magical Papyri », in SCHÄFER - KIPPENBERG (1997), p. 65-92.

Graf, F., Gottesnähe und Schadenzauber. Die Magie in der griechisch-römischen Antike, München, Beck, 1996.

Grandet, P., Contes de l'Égypte ancienne, Paris, 1998.

Griffith, F.L., Stories of the High Priests of Memphis, vol. I, Oxford, 1900.

—, Thompson, H., The Demotic Magical Papyrus of London and Leiden, 3 vols., London, 1904-1909. 
HopfNER, Th. (1921-24), Griechisch-Ägyptischer Offenbarungszauber, I Leipzig 1921, reimpr. Amsterdam, A.M. Hakkert, 1974; II1 Leipzig 1924, reimpr. Amsterdam, A.M. Hakkert, 1983; II2 Leipzig 1924, reimpr. Amsterdam, A.M. Hakkert, 1990.

Johnston, S. I., Ancient Greek Divination, Oxford, Wiley-Blackwell, 2008.

Jordan, D., «Two Papyri with Formulae for Divination », in MireCKI - Meyer (2002), p. 25-26.

Koenig, Y., Magie et magiciens dans l'Égypte ancienne, Paris, Pygmalion-Gérard Watelet, 1994.

- (éd.), La magie en Égypte : à la recherche d'une définition, Paris, La documentation Française, 2002.

-, «Conclusion. La magie égyptienne : pour une meilleure prise en compte de la magie », in Koenig (2002), p. 397-414.

LEClant, P., «Éléments pour une étude de la divination dans l'Égypte pharaonique », in A. CAQUOT, M. Leibovici (éd.), La divination, vol. I, Paris, PUF, 1968, p. 1-23.

LeFÈBvre, G., Romans et contes égyptiens de l'époque pharaonique, Paris, Maisonneuve, 1948.

LEWIS, N., «A Ban on False Prophets: Pap. Coll. Youtie 30 », CE 52, 103 (1977), p. 143-146.

Merkelbach, R. (ed.), Abrasax. Ausgewäblte Papyri Religiösen und Magischen Inhalts, Band 3: Zwei griechisch-ägyptische Weiherzeremonien (Die leidener Weltschöpfung - Die Pschai-Aion-Liturgie), Opladen, 1992 (Papyrologica coloniensia, vol XVII).

—, Isis Regina, Zeus Sarapis. Die griechisch-ägyptische Religion nach den Quellen dargestellt, Stuttgart/ Leipzig, Teubner, 1995.

Mirecki, P., MeYer, M., Magic and Ritual in the Ancient World, Leiden, Brill, 2002.

Monaca, M. (éd.), Problemi di Storia Religiosa del Mondo Tardo-Antico. Tra Mantica e Magia, Cosenza, Edizioni Lionello Giordano, 2009.

MoYer, I.S., «The Initiation of the Magician. Transition and Power in Graeco-Egyptian Ritual », in DodD - Faraone (2003), p. 219-238.

—, "Thessalos of Tralles and Cultural Exchange», in Noegel - Walker - WheELER (2003), p. 39-56

—, Egypt and the Limits of Hellenism, Cambridge, CUP, 2011

Noegel, S., Walker, J., Wheeler, B., Prayer, Magic and the Stars in the Ancient and Late Antique World, Pennsylvania State University, 2003.

Ogden, D., In Search of the Sorcerer's Apprentice. The Traditional Tales of Lucian's Lover of Lies, Swansea, The Classical Press of Wales, 2007.

ParÁssoglou, G.M., «Circular from a Prefect: Sileat Omnibus Perpetuo Divinandi Curiositas », in Collectanea Papyrologica (Texts Published in Honor of A.C. Youtie), edited by Ann Ellis Hanson, Bonn, R. Habelt, 1976, p. 261-274.

PINCH, G., Magic in Ancient Egypt, University of Texas, $2009^{2}$ (1 $1^{\text {re }}$ éd. London, British Museum Press, 1994; $1^{\text {re }}$ éd. dans University of Texas Press, 2006).

Quaegebeur, J., «La désignation P3Hry-ip: PHRITOB », in J. Osing, G. Dreyer (éd.), Form und Mass. Beiträge zur Literatur, Sprache und Kunst des Alten Ägytten (Festschrift für Gerhardt Fecht), Wiesbaden, 1987, p. 368-394.

—, «L'appel au divin : le bonheur des hommes dans la main des dieux », in J.-G. HeINTZ (éd.), Oracles et prophéties dans l'Antiquité, Paris, de Boccard, 1997 (Travaux du Centre de Recherche sur le Proche Orient, 15), p. 15-34.

Reitzenstein, R., Poimandres, Berlin, Teubner, 1905.

RitNer, R.K., "Egyptian Magical Practice under the Roman Empire: the Demotic Spells and their Religious Context", $A N R W$ II 18.5, Berlin and New York, 1995, p. 3333-3379.

—, « Necromancy in Ancient Egypt», in CiraOlo - Seidel (2002a), p. 89-96.

—, «Des preuves de l'existence d'une nécromancie dans l'Égypte ancienne », in KoeNIG (2002), p. 285-306.

-, The Mechanics of Ancient Egyptian Magical Practice, Chicago, The Oriental Institute of the University of Chicago, 20084. 
SAuneron, S., Les prêtres de l'ancienne Égypte, Paris, Seuil, 1998 (1 ${ }^{\text {re }}$ éd. 1957).

—, «Les songes et leur interprétation dans l'Égypte ancienne », in Les songes et leur interprétation, Paris, Éd. du Seuil, 1959 (Sources orientales, 2), p. 17-61.

—, «Asclepio divinità epifanica e salvatrice: l'esperienza di Thessalos fra medicina, religione e magia ", in E. De Miro, G. Sfameni Gasparro, V. Calì (éd.), Il culto di Asclepio nell'area mediterránea (Atti del Convegno Internazionale Agrigento 20-22 novembre 2005), Roma, 2010, p. 287-311.

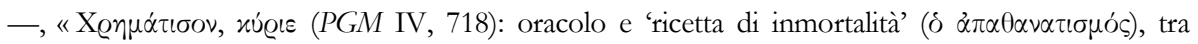
religione filosofica, mistero e magia », MHNH 11 (2011), p. 83-122.

Sмith, J.Z., Map is not Territory. Studies in the History of Religions, Leiden, Brill, 1978.

Suárez de la Torre, E., Oráculos sibilinos, in A. Díez Macho, A. Piñero (éd.), Apócrifos del Antiguo Testamento, Madrid, Cristiandad, 2002², vol. III, p. 331-603 (1 $\left.{ }^{\mathrm{a}} \mathrm{ed} .1982\right)$.

—, "Forme e funzioni del fenómeno profetico e divinatorio dalla Grecia arcaica al periodo tardoantico ", in G. SFAMENI GASPARro et al. (éd.), Modi di comunicazione tra il divino e l'umano, Cosenza, Edizioni Lionello Giordano, 2005, p. 29-106 (collana Hiera, nº 7).

—, « La divinazione nei papiri magici greci «, in MONACA (2009b), p. 13-44.

—, "Versos homéricos en los papiros mágicos griegos », in M.J. GARCía BlanCO et al. (éd.),

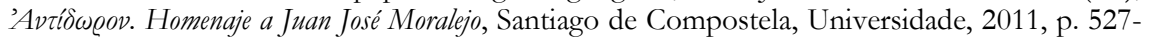
544.

—, «Pseudepigraphy and Magic », in J. MARTínez (éd.), Fakes, Forgeries \& Issues of Authenticity in Classical Literature, Cambridge 2013a.

—, « Mito, teología, magia y astrología en PGM XIII (P.Leid. I 395) », in E. SuÁreZ DE LA TORRE, A. Pérez Jiménez (éd.), Mito y Magia en Grecia y Roma, Barcelona, 2013b (sous presse).

SzPakowskA, K., Behind Closed Eyes: Dreams and Nightmares in Ancient Egypt, Classical Press of Wales, 2003a.

—, «The Open Portal. Dreams and Divine Power in Pharaonic Egypt», in Noegel - Walker WHEELER (2003), p. 111-124 [= SZPAKOWSKA (2003b)]

- (éd.), Through a Glass Darkly. Magic, Dreams, and Prophecy in Ancient Egypt, Swansea, The Classical Press of Wales, 2006.

Van der Horst, W. (éd.), Chaeremon. Egyptian Priest and Stoic Philosopher, Leiden, Brill, 1984.

ZAGO, M., La ricetta di immortalitá, Milano, Vita Felice, 2010. 